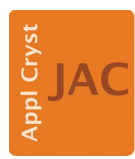

JOURNAL OF

APPLIED

CRYSTALLOGRAPHY

ISSN 1600-5767

Received 24 December 2015

Accepted 1 April 2016

Edited by T. White, Center for Free-Electron Laser Science, Hamburg, Germany

1 This article will form part of a virtual special issue of the journal on free-electron laser software.

Keywords: serial crystallography; outlier identification; $\mathrm{CC}_{1 / 2}$; precision; model bias; isomorphism; non-isomorphism.

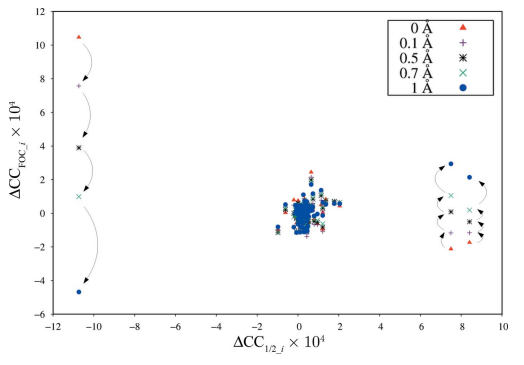

OPEN $\odot$ ACCESS

\section{Identification of rogue datasets in serial crystallography}

\author{
Greta Assmann, Wolfgang Brehm and Kay Diederichs*
}

Department of Biology, University of Konstanz, Box 647, Konstanz, D-78457, Germany. *Correspondence e-mail: kay.diederichs@uni-konstanz.de

Advances in beamline optics, detectors and X-ray sources allow new techniques of crystallographic data collection. In serial crystallography, a large number of partial datasets from crystals of small volume are measured. Merging of datasets from different crystals in order to enhance data completeness and accuracy is only valid if the crystals are isomorphous, i.e. sufficiently similar in cell parameters, unit-cell contents and molecular structure. Identification and exclusion of non-isomorphous datasets is therefore indispensable and must be done by means of suitable indicators. To identify rogue datasets, the influence of each dataset on $\mathrm{CC}_{1 / 2}$ [Karplus \& Diederichs (2012). Science, 336, 1030-1033], the correlation coefficient between pairs of intensities averaged in two randomly assigned subsets of observations, is evaluated. The presented method employs a precise calculation of $\mathrm{CC}_{1 / 2}$ that avoids the random assignment, and instead of using an overall $\mathrm{CC}_{1 / 2}$, an average over resolution shells is employed to obtain sensible results. The selection procedure was verified by measuring the correlation of observed (merged) intensities and intensities calculated from a model. It is found that inclusion and merging of non-isomorphous datasets may bias the refined model towards those datasets, and measures to reduce this effect are suggested.

\section{Introduction}

Several bottlenecks hamper structure determination of biological macromolecules. One practical problem is often the lack of suitably large crystals for collection of complete highresolution data, as the diffraction signal for a given incident $\mathrm{X}$-ray beam is proportional to the well ordered crystal volume illuminated by the beam, as given by Darwin's formula (Darwin, 1914, 1922; Blundell \& Johnson, 1976). Smaller crystals require higher X-ray beam intensities to produce diffraction up to the same resolution as comparable crystals with larger volume, but because of radiation damage do not result in complete datasets.

This problem of incompleteness has been addressed by combining several partial datasets from multiple crystals in order to obtain a complete dataset averaged ('merged') over all observations of every unique reflection. Merging of data from a few (2-20) crystals has been standard practice in crystallography (Kendrew et al., 1960; Dickerson et al., 1961), but recently this concept was extended to tens or even thousands of crystals with only a few reflections per dataset and termed serial crystallography (SX). When using extremely short pulses of X-rays, each of tens of femtoseconds in duration, generated by a free-electron laser (FEL) the method is referred to as serial femtosecond crystallography (SFX; Chapman et al., 2011). This also exploits the 'diffraction before destruction' approach, where single diffraction 'snapshots' are collected before radiation damage can occur. Serial crystal- 
lography performed at the synchrotron (SSX; Rossmann, 2014) is a more recent development based on data collection and processing methods established for single-crystal work, but enhanced by procedures for crystal identification by scanning crystallization plates using conventional optics or $\mathrm{X}$-rays. This approach is also ideally suited for employing novel crystallization setups such as the lipidic cubic phase and in situ data collection at room or cryo temperature (Huang et al., 2015, 2016).

Although serial crystallography, especially SFX, can in principle solve or mitigate the problem of radiation damage and the lack of sufficiently large crystals, one potential obstacle is non-isomorphism of crystals. In order to merge different partial datasets into one complete dataset, one must ensure that all partial datasets are sufficiently isomorphous. Isomorphous crystals are structurally identical and correspond to the same atomic model such that datasets only differ by random error, whereas non-isomorphous crystals represent different structural features, at the level of cell parameters, composition (in terms of presence of molecular entities like ligands and solvent molecules) or molecular conformation. The variation between datasets thus also depends on the extent of non-isomorphism.

To group datasets on the basis of similarity, hierarchical clustering based on pairwise correlation coefficients was employed by Giordano et al. (2012). The basic idea here is that a low correlation coefficient indicates unrelatedness of datasets, which is interpreted as non-isomorphism. This method may also falsely reject datasets with a high level of random error, or in other words, weak datasets. Essentially, this would trade accuracy for precision.

Another method that can be employed is hierarchical clustering of datasets based on their cell parameters (Foadi $e t$ al., 2013). This method avoids the problem of false positive rejection of weak datasets, but on the other hand similarity of cell parameters is only a necessary but not a sufficient condition, and does not take similarity of diffraction into account. The criterion can therefore be considered as a rather weak filter.

Yet another approach combines the unit-cell changes, the intensity correlation coefficient and a relative anomalous correlation coefficient for clustering of datasets (Liu et al., 2013).

Following previous work (Karplus \& Diederichs, 2012; Diederichs \& Karplus, 2013), we chose the numerical value of $\mathrm{CC}_{1 / 2}$, an indicator for the precision of the data resulting from merging of the partial datasets, as an optimization target. We have shown earlier that $\mathrm{CC}_{1 / 2}$ limits - as seen from the properties of the derived quantity $\mathrm{CC}^{*}$ - the ability to obtain good agreement between model and data (Karplus \& Diederichs, 2012). Since the goal of structure analysis is to obtain a model consistent with the data, it appears logical to optimize $\mathrm{CC}_{1 / 2}$.

$\mathrm{CC}_{1 / 2}$ can be evaluated and optimized as a function of the datasets being merged, and we propose and study a procedure to identify non-isomorphous crystals from multi-crystal datasets based on their influence on $\mathrm{CC}_{1 / 2}$. Simulated data as well as experimental datasets from two projects, PepT and AlgE
Table 1

Crystallographic statistics of experimental datasets.

\begin{tabular}{|c|c|c|}
\hline & РepT & AlgE \\
\hline PDB code & $4 \times n i$ & $4 \times n k$ \\
\hline Space group & (20) $C 222_{1}$ & (19) $P 2_{1} 2_{1} 2_{1}$ \\
\hline Unit-cell parameters $(\AA)$ & $\begin{array}{l}a=106.88, b=106.88 \\
\quad c=111.14\end{array}$ & $\begin{array}{c}a=48.01, b=74.34, \\
\quad c=184.69\end{array}$ \\
\hline Wavelength $(\AA)$ & 0.979180 & 1.033000 \\
\hline No. of crystals & 159 & 266 \\
\hline Resolution (§) & $50-2.78$ & $50-2.54$ \\
\hline Completeness (\%) & 97.6 & 84.7 \\
\hline $\begin{array}{l}\text { Completeness highest resolu- } \\
\text { tion shell }\end{array}$ & $67.6(2.85-2.78)$ & $7.6(2.61-2.54)$ \\
\hline Total No. of observations & 905207 & 151228 \\
\hline $\begin{array}{l}\text { No. of observations per crystal } \\
\text { (min-max, mean) }\end{array}$ & $2592-6040,5693$ & $59-507,564$ \\
\hline No. of unique reflections & 16485 & 18684 \\
\hline$R_{\text {meas }}$ & 0.973 & 0.565 \\
\hline $\mathrm{CC}_{1 / 2}$ & 0.992 & 0.926 \\
\hline$\langle I / \sigma(I)\rangle$ & 4.25 & 2.74 \\
\hline
\end{tabular}

(Huang et al., 2015), were analysed to identify non-isomorphous datasets.

\section{Methods}

\subsection{Simulated data}

Eleven complete datasets to $1.46 \AA$ resolution were simulated with $S I M \_M X$ (Diederichs, 2009) using the atomic coordinates of cubic insulin [Protein Data Bank (PDB) code 2 bn3 (Nanao et al., 2005); space group 1233 ] and a flat bulk solvent (density $0.35 \mathrm{e}^{-} \mathrm{A}^{-3}$ and $B=50 \AA^{2}$ ) for the calculation of structure factors. To simulate a specific case of nonisomorphism by numerical experiments, the unit-cell parameters were elongated by different amounts $(0-1 \AA)$. This results in a different sampling of the molecular transform and thus changes the intensities. Artificial $F_{\text {calc }}^{2}$ to be used as intensities were then calculated using PHENIX.FMODEL (Adams et al., 2002), and simulated raw datasets were calculated with SIM_MX and processed with XDS (Kabsch, 2010a,b). Different random seeds ensured that different (pseudo-) random errors for different datasets were calculated.

\subsection{Experimental data}

Crystallization and data collection of the membrane protein peptide transporter PepT from Streptococcus thermophilus (483 residues) and AlgE, the alginate transporter from Pseudomonas aeruginosa (490 residues), were described previously (Huang et al., 2015). PepT and AlgE X-ray diffraction data consisting of small rotation wedges of different crystals, to 2.78 and $2.54 \AA$ resolution, respectively, were collected at room temperature from crystals in meso and in situ at the PX II beamline of the Swiss Light Source (Villigen, Switzerland). Each rotation wedge was collected from a different crystal and is denoted as a (partial) dataset in the following. Data were processed with $X D S$ and XSCALE (Kabsch, 2010a,b). For this work, not all datasets that were used at the time of PDB deposition were available; thus for PepT, we used 159, and for 
AlgE, we used all 266 datasets available, 175 of which were used in the work of Huang et al. (2015). Table 1 summarizes these data.

\subsection{Calculation of $\mathrm{CC}_{1 / 2}$ : the $\sigma-\tau$ method}

For the calculations of $\mathrm{CC}_{1 / 2}$ the XSCALE output file (XSCALE. HKL) was used. Merged intensities of observations were weighted with their sigma values as assigned by the data processing programs, $X D S$ and $X S C A L E$.

As defined by Karplus \& Diederichs (2012), $\mathrm{CC}_{1 / 2}$ can be calculated from the formula for Pearson's correlation coefficient:

$$
\mathrm{CC}_{1 / 2}=\frac{\sum\left(a_{i}-\bar{a}\right)\left(b_{i}-\bar{b}\right)}{\left[\sum\left(a_{i}-\bar{a}\right)^{2} \sum\left(b_{i}-\bar{b}\right)^{2}\right]^{1 / 2}},
$$

where $a_{i}$ and $b_{i}$ are the intensities of unique reflections merged across the observations randomly assigned to subsets $\mathrm{A}$ and $\mathrm{B}$, respectively, and $\bar{a}$ and $\bar{b}$ are their averages.

For this work, we calculated $\mathrm{CC}_{1 / 2}$ in a different way, avoiding the random assignment to subsets. $\mathrm{CC}_{1 / 2}$ may be expressed as (Karplus \& Diederichs, 2012, supplement)

$$
\mathrm{CC}_{1 / 2}=\frac{\sigma_{\tau}^{2}}{\sigma_{\tau}^{2}+\sigma_{\varepsilon}^{2}},
$$

with

$\tau=$ difference between true values of intensities and their average (thus $\tau$ has zero mean),

$\varepsilon_{\mathrm{A}, \mathrm{B}}=$ random errors in merged intensities of half-sized subsets (with zero mean) which are mutually independent and on average of equal magnitude,

$\sigma_{\tau}^{2}=$ variance of $\tau$,

$\sigma_{\varepsilon}^{2}=$ variance of $\varepsilon_{\mathrm{A}, \mathrm{B}}$.

Then, the full dataset merged intensity $y$ (after subtraction of the average) is $\tau+\left(\varepsilon_{\mathrm{A}}+\varepsilon_{\mathrm{B}}\right) / 2$ and

$$
\sigma_{y}^{2}=\sigma_{\tau}^{2}+\frac{1}{2} \sigma_{\varepsilon}^{2} .
$$

We may thus substitute $\sigma_{y}^{2}-\frac{1}{2} \sigma_{\varepsilon}^{2}$ for $\sigma_{\tau}^{2}$ and obtain

$$
\mathrm{CC}_{1 / 2}=\frac{\sigma_{\tau}^{2}}{\sigma_{\tau}^{2}+\sigma_{\varepsilon}^{2}}=\frac{\left(\sigma_{y}^{2}-\frac{1}{2} \sigma_{\varepsilon}^{2}\right)}{\left(\sigma_{y}^{2}-\frac{1}{2} \sigma_{\varepsilon}^{2}\right)+\sigma_{\varepsilon}^{2}}=\frac{\left(\sigma_{y}^{2}-\frac{1}{2} \sigma_{\varepsilon}^{2}\right)}{\left(\sigma_{y}^{2}+\frac{1}{2} \sigma_{\varepsilon}^{2}\right)} \text {. }
$$

This just requires calculation of $\sigma_{y}^{2}$, the variance of the average intensities across the unique reflections of a resolution shell, and $\frac{1}{2} \sigma_{\varepsilon}^{2}$, the average variance of the observations contributing to the merged intensities.

As shown above, this ' $\sigma-\tau$ method' of $\mathrm{CC}_{1 / 2}$ calculation is mathematically equivalent to the calculation of a Pearson correlation coefficient for the special case of two sets of values (intensities) that randomly deviate from their common 'true' values. Since it avoids the random assignment into half-datasets, it is not influenced by any specific random number sequence and thus yields more consistent values, as further discussed below ( $\$ 4.1)$.

The average intensity of macromolecular diffraction data diminishes with increasing resolution. If all data, from low to high resolution, are used for calculation of an overall $\mathrm{CC}_{1 / 2}$, the resulting correlation coefficient is dominated by the strong low-resolution terms and therefore biased towards large positive values; the overall $\mathrm{CC}_{1 / 2}$ is thus almost meaningless (Karplus \& Diederichs, 2015). More meaningful $\mathrm{CC}_{1 / 2}$ values are obtained when dividing the data into (usually ten or more) resolution shells, since in each resolution shell the average intensity can be considered constant. To obtain a single value, we average the $\mathrm{CC}_{1 / 2}$ values obtained in all resolution shells, while weighting with the number of contributing reflections.

\subsection{The $\Delta \mathrm{CC}_{1 / 2}$ method}

Since our goal is to maximize $\mathrm{CC}_{1 / 2}$ by excluding datasets, we used the following simple algorithm that avoids any combinatorial explosion of possibilities. We define as $\mathrm{CC}_{1 / 2 \text { _overall }}$ the value of the average $\mathrm{CC}_{1 / 2}$ when all datasets are included for calculation. Furthermore, we denote as $\mathrm{CC}_{1 / 2 \_i}$ the value of $\mathrm{CC}_{1 / 2}$ when all datasets are included except for one dataset $i$, which is omitted from calculations. $\mathrm{CC}_{1 / 2 \_i}$ is calculated for every dataset $i$. We define

$$
\Delta \mathrm{CC}_{1 / 2_{-} i}=\mathrm{CC}_{1 / 2_{-} i}-\mathrm{CC}_{1 / 2_{-} \text {overall }} .
$$

If $\Delta \mathrm{CC}_{1 / 2 \_i}>0(<0)$, this dataset is improving (impairing) the overall $\mathrm{CC}_{1 / 2}$. After rejection of the dataset belonging to the worst negative $\triangle \mathrm{CC}_{1 / 2 \_}$, all remaining datasets are processed by XSCALE again, because any dataset influences all scale factors. The newly scaled output file can be used to identify another potential non-isomorphous dataset, and this may be iterated until no further significant improvement is obtained; usually, two or three iterations are sufficient.

\subsection{Validation of isomorphous dataset selection}

We devised a strategy to assess an actual improvement of the data by comparison with the squared $F_{\text {calc }}$ moduli obtained from a structural model. To this end, deposited PDB models (4xnj for PepT, 4xnk for AlgE; Huang et al., 2015) were processed with PHENIX.FMODEL to produce $F_{\text {calc }}^{2}$ reference data, to be used for comparison with $I_{\text {obs. }}$ We define $\mathrm{CC}_{\mathrm{FOC} \_ \text {overall }}$ as the correlation coefficient of observed and calculated intensities when all datasets are included. Moreover, we define $\mathrm{CC}_{\mathrm{FOC} \_i}$ as the correlation coefficient with all datasets included except for one dataset $i$, which is omitted from calculations. The difference

$$
\Delta \mathrm{CC}_{\mathrm{FOC}_{-} i}=\mathrm{CC}_{\mathrm{FOC}_{-} i}-\mathrm{CC}_{\mathrm{FOC}_{-} \text {overall }}
$$

then gives the improvement or impairment the dataset $i$ causes in the overall correlation of data and model. $\Delta \mathrm{CC}_{\mathrm{FOC}_{-} i}<0$ indicates an impairment in the similarity of data and model; $\Delta \mathrm{CC}_{\mathrm{FOC}_{-} i}>0$ indicates an improvement. We chose to compare $\Delta \mathrm{CC}_{\mathrm{FOC}_{\_} i}$ and $\Delta \mathrm{CC}_{1 / 2 \_i}$ although it would be more appropriate to use CC* (Karplus \& Diederichs, 2012) instead of $\mathrm{CC}_{1 / 2}$, or in other words, to compare $\Delta \mathrm{CC}_{\mathrm{FOC}_{-} i}$ and a quantity defined analogously to $\Delta \mathrm{CC}_{1 / 2 \_i}, \Delta \mathrm{CC}_{i}^{*}=\mathrm{CC}_{i}^{*}-$ $\mathrm{CC}_{\text {overall }}^{*}$. However, since $\mathrm{CC}^{*}$ depends monotonically on $\mathrm{CC}_{1 / 2}$, any qualitative finding obtained in a comparison of $\Delta \mathrm{CC}_{\mathrm{FOC}_{\_} i}$ and $\Delta \mathrm{CC}_{1 / 2 \_i}$ would be the same as for a $\Delta \mathrm{CC}_{\mathrm{FOC}_{\_} i}$ and $\Delta \mathrm{CC}_{i}^{*}$ comparison. 
Table 2

$\Delta \mathrm{CC}_{1 / 2 \_i}$ of synthetic datasets with elongated unit-cell parameters.

\begin{tabular}{ll}
\hline Change of unit-cell parameters $(\AA)$ & $\Delta \mathrm{CC}_{1 / 2 \_} i$ \\
\hline+1.0 & -0.518 \\
+0.8 & -0.313 \\
+0.6 & -0.271 \\
+0.4 & -0.262 \\
+0.2 & +0.873 \\
+0.1 (2 datasets) & $+0.785,+0.785$ \\
0.0 (4 datasets) & $+0.710,+0.706,+0.698,+0.684$ \\
\hline
\end{tabular}

For some calculations, random shifts of atom coordinates of the original PDB file were introduced by MOLEMAN2 (Kleywegt, 1995).

\section{Results}

\subsection{Characteristics of $\Delta \mathbf{C C}_{1 / 2}$ for the simulated data}

Eleven complete datasets with different changes in the unitcell parameters were used to simulate a realistic case where most of the datasets are isomorphous relative to each other but some are (non-isomorphous) outliers.

The $\Delta \mathrm{CC}_{1 / 2}$ method was applied to the simulated datasets. In general, increasing changes in unit-cell parameters are associated with decreasing $\Delta \mathrm{CC}_{1 / 2 \_i}$, as expected (Table 2). The largest change in unit-cell parameters $(1.0 \AA)$ shows the lowest $\Delta \mathrm{CC}_{1 / 2 \_i}$, which is thus correctly identifying nonisomorphism. $\Delta \mathrm{CC}_{1 / 2 \_i}$ shows highly positive values for those datasets where no or only slight changes were introduced (0.0$0.2 \AA) . \Delta C_{1 / 2 \_i}$ does not increase linearly; rather, it drops dramatically from 0.2 to $0.4 \AA$. On the basis of $\Delta \mathrm{CC}_{1 / 2 \_}$, the most isomorphous dataset is the one with a $0.2 \AA$ cell parameter change; this appears to be a sensible result since its intensities are the most closely related to those of all other datasets.

The numerical values of $\Delta \mathrm{CC}_{1 / 2 \_i}$ change to a much greater extent $(-0.5$ to 0.8$)$ than in the experimental case. This is because the impact of one complete dataset (out of 11) is high in comparison to a single small rotation wedge SSX dataset (out of hundreds). Moreover, the artificially induced gap between nearly perfect isomorphous datasets (cell changes of $0.0-0.4 \AA$, i.e. close to the average of all cell changes) and very few severely non-isomorphous datasets (cell changes of 0.6$1.0 \AA$ ) enforces this effect.

\subsection{PepT: model unbiased by non-isomorphous dataset}

For PepT, 159 datasets were analysed, as seen in Fig. 1, where a histogram of $\Delta \mathrm{CC}_{1 / 2 \_i}$ is shown. The histogram is dominated by a Gaussian-shaped central part slightly above $\Delta \mathrm{CC}_{1 / 2}=0$, with standard deviation $\sigma=1.68 \times 10^{-4}$. Datasets with a $\Delta \mathrm{CC}_{1 / 2 \_i}$ of around zero do not significantly change the overall $\mathrm{CC}_{1 / 2}$; however, their inclusion is necessary for increased completeness and multiplicity. One dataset has a significantly $(-28.8 \sigma)$ negative $\Delta \mathrm{CC}_{1 / 2 \_i}$ and is thus identified as non-isomorphous. Some datasets have, at $31.8 \sigma$ and $76.2 \sigma$, a highly positive $\Delta \mathrm{CC}_{1 / 2 i}$; they significantly decrease $\mathrm{CC}_{1 / 2 \text { overall }}$ if rejected and appear to be particularly valuable.

Comparing, in terms of raw data appearance and processing logfiles and statistics reported by XDS or XSCALE, positive or negative outlier datasets with datasets from the central part of the histogram showed no striking peculiarities for any of the analysed criteria such as number of reflections or similar. In particular, negative $\Delta \mathrm{CC}_{1 / 2 \_i}$ was not predictable from unitcell parameters, spot shape or other data processing statistics of single datasets. Furthermore, the negative outlier had not been identified by the ISa-based (Diederichs, 2009) rejection of datasets performed by Huang et al. (2015). However, we note that important experimental variables, like crystal volume, are not recorded during the experiments.

Fig. 2 shows a plot of $\Delta \mathrm{CC}_{\mathrm{FOC}_{-} i}$ against $\Delta \mathrm{CC}_{1 / 2 \_i}$ for the datasets of this project. If our procedure for identifying nonisomorphism is meaningful, we expect an improvement of the correlation between model $F_{\text {calc }}^{2}$ and merged $I_{\text {obs }}$ for those datasets that increase $\mathrm{CC}_{1 / 2}$, and a decrease of correlation for the non-isomorphous ones. As could be expected from the

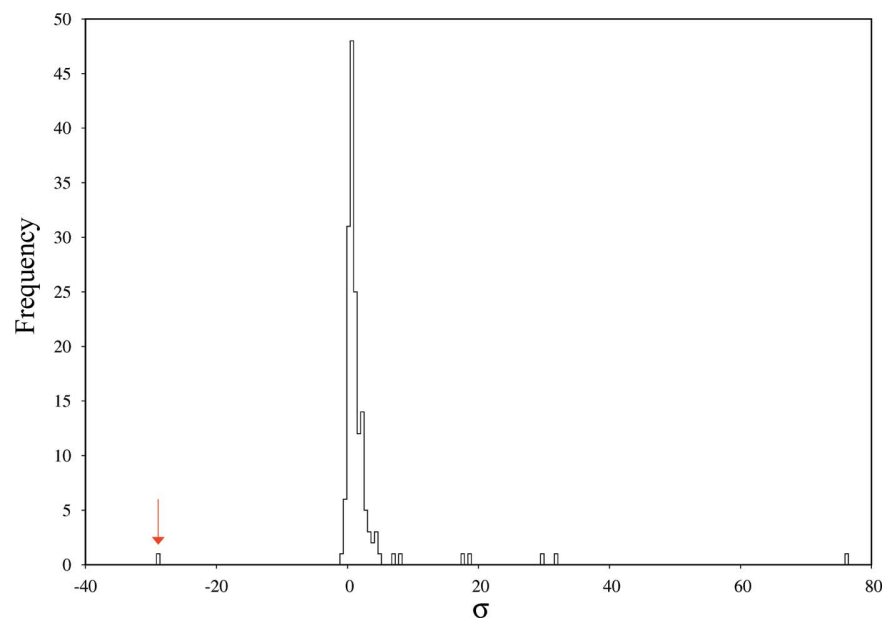

Figure 1

Histogram of $\Delta \mathrm{CC}_{1 / 2 \_i}$ values for PepT. The $-28.8 \sigma$ unit outlier is indicated with an arrow.

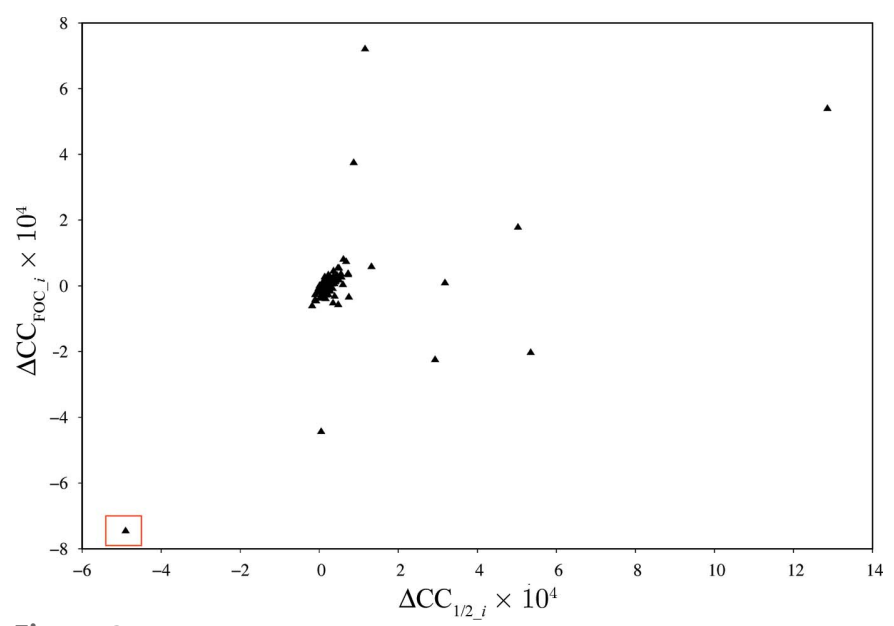

Figure 2

Plot of $\Delta \mathrm{CC}_{\mathrm{FOC} i}$ against $\Delta \mathrm{CC}_{1 / 2 i}$ for PepT. The $-28.88 \sigma$ unit outlier $\left(\Delta \mathrm{CC}_{1 / 2 \_i} \simeq-4.8 \times 10^{-4}\right)$ is boxed. 
histogram of Fig.1, most of the data sets cause little change of $\Delta \mathrm{CC}_{\mathrm{FOC}}$ and $\Delta \mathrm{CC}_{1 / 2}$; they cluster in the middle of the diagram. The dataset identified as the most non-isomorphous one indeed shows the worst correlation of experimental data and model; $\mathrm{CC}_{\mathrm{FOC}}$ is significantly improved when rejecting this specific dataset. Conversely, some of the datasets show a clear improvement of $\mathrm{CC}_{\mathrm{FOC}}$ and $\mathrm{CC}_{1 / 2}$.

The validation appears to work satisfactorily despite the fact that the $4 \mathrm{xnj}$ model derived from cryo data and used here for validation is itself not isomorphous with the data, since the cell parameters of the cryo and the room-temperature crystals differ in $a$ and $b$ by about $4 \%$.

\subsection{AlgE: model biased by non-isomorphous dataset}

AlgE displays a similar histogram $\left(\sigma=7.22 \times 10^{-4}\right)$ of $\Delta \mathrm{CC}_{1 / 2 \_}$values (Fig. 3) as PepT. The worst non-isomorphous dataset is, at $-14.8 \sigma$ units, an obvious outlier of the $\Delta \mathrm{CC}_{1 / 2} i$ distribution. Similarly, positive outliers exist at $10.1 \sigma$ and $11.5 \sigma$ units. As for PepT, we did not observe in the raw data or in the processing and scaling statistics any particular properties of positive or negative outliers. Again, the strongest negative outlier had not been identified by the ISa-based rejection of datasets performed by Huang et al. (2015).

In contrast to Fig. 2, the plot of $\Delta \mathrm{CC}_{\mathrm{FOC}_{-} i}$ against $\Delta \mathrm{CC}_{1 / 2 \_} i$ for $\mathrm{AlgE}$ shows an unexpected behaviour (Fig. 4). As expected, most of the datasets cluster at $\Delta \mathrm{CC}_{1 / 2}$ and $\Delta \mathrm{CC}_{\mathrm{FOC}}$ values around zero, but the dataset identified by $\Delta \mathrm{CC}_{1 / 2 \_i}$ as the most non-isomorphous dataset is surprisingly showing the best $\triangle \mathrm{CC}_{\mathrm{FOC}_{\_} i}$. Conversely, the best datasets as judged from $\Delta \mathrm{CC}_{1 / 2 \_i}$ exhibit negative $\Delta \mathrm{CC}_{\mathrm{FOC}_{\_} i}$.

After some investigation, we attributed these observations to our choice of PDB models used for validation. In the case of PepT, we had chosen the model based on an independent single-crystal cryo dataset (4xnj), whereas in the case of $\mathrm{AlgE}$, we had chosen 4 xnk which had been refined against those datasets we were now trying to characterize. In the case of AlgE, refinement of the model 4xnk against the SSX data had led to a bias in the sense that the model partly fits the systematic effects contributing to the non-isomorphism of the worst dataset. This bias additionally leads to a reduced $\Delta \mathrm{CC}_{\mathrm{FOC}_{-} i}$ since the model is biased into a state more distant from those of datasets with positive $\Delta \mathrm{CC}_{1 / 2 \_i}$.

We therefore performed an additional experiment: By applying random shifts of magnitude up to $1.0 \AA$ to the $4 x n k$ coordinates, we attempted to 'shake' the model out of its local minimum. Recalculating $\Delta \mathrm{CC}_{\mathrm{FOC}_{-} i}$ for increasing shifts, we progressively observed the expected behaviour of a positive correlation between $\Delta \mathrm{CC}_{1 / 2 \_i}$ and $\Delta \mathrm{CC}_{\mathrm{FOC}_{\_} i}$, as emphasized by arrows in Fig. 4. This is most pronounced for the dataset with largest negative $\Delta \mathrm{CC}_{1 / 2}$, which is seen to have strongly negative $\Delta \mathrm{CC}_{\mathrm{FOC}_{-} i}$ when the model is shaken most. However, the datasets with most positive $\Delta \mathrm{CC}_{1 / 2 \_i}$ did not fully reach high values of $\Delta \mathrm{CC}_{\mathrm{FOC}_{-} i}$, presumably because, for the highest shifts, the model is strongly degraded so that it cannot fit the data well.

Ideally, validation is done with a model that is independent of the data. Another experiment was therefore performed

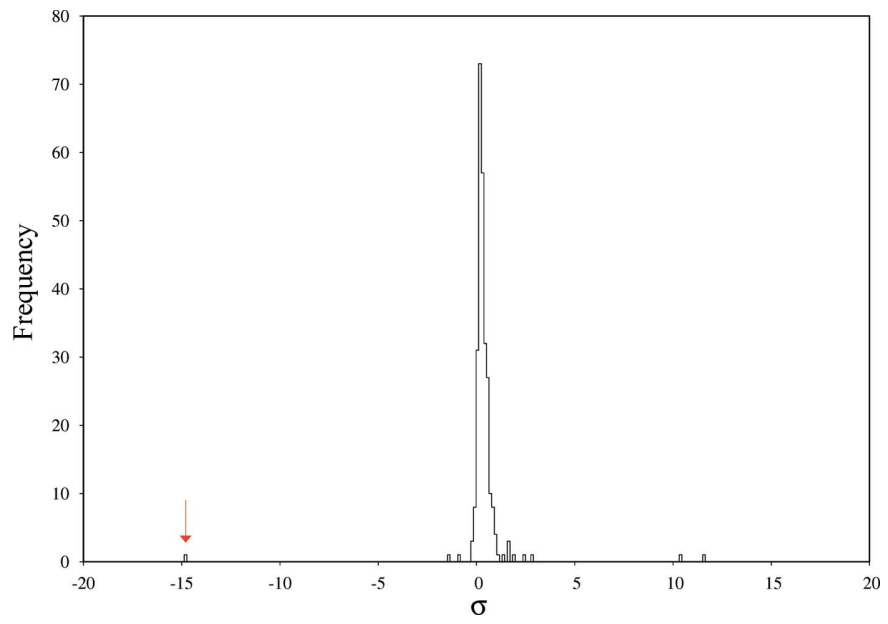

Figure 3

Histogram of $\Delta \mathrm{CC}_{1 / 2 \_i}$ values for $\mathrm{AlgE}$. The $-14.8 \sigma$ unit outlier is indicated with an arrow.

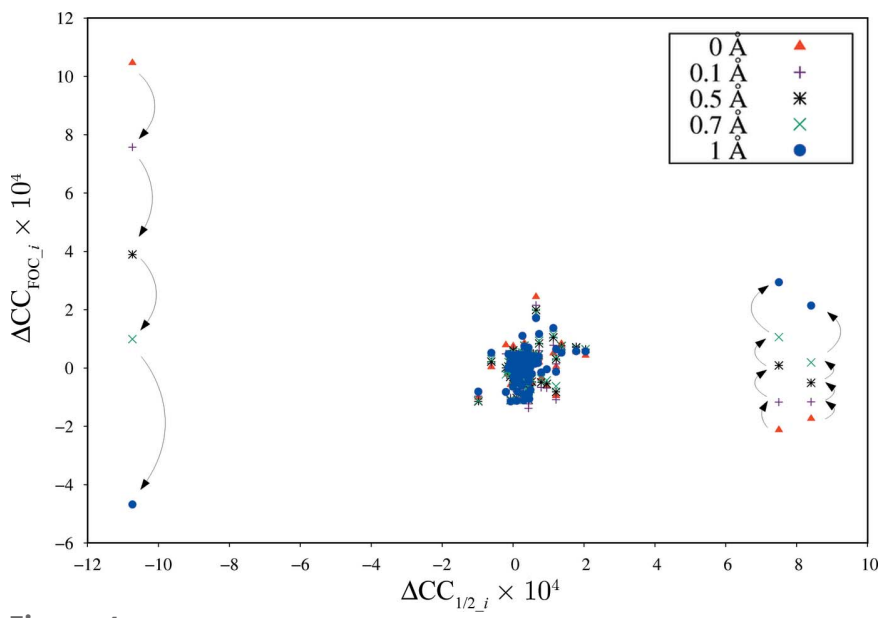

Figure 4

Plot of $\Delta \mathrm{CC}_{\mathrm{FOC}_{-} i}$ against $\Delta \mathrm{CC}_{1 / 2 i i}$ for AlgE. Different colours and marker symbols refer to the different random shifts of the atom coordinates. Arrows indicate the change of $\Delta \mathrm{CC}_{\mathrm{FOC}_{-} i}$ upon increasing the magnitude of random shifts for the three most significant outliers of the Gaussian distribution of Fig. 3.

with the $4 \times n$ coordinates derived from a dataset collected at cryo temperature. In this case, no bias is present, and indeed there is a positive correlation between $\Delta \mathrm{CC}_{1 / 2 \_} i$ and $\Delta \mathrm{CC}_{\mathrm{FOC} \_i}$ (data not shown), similar to Fig. 2 for PepT, as expected.

\section{Discussion}

\subsection{Calculation of $\mathrm{CC}_{1 / 2}$}

We have shown above that $\mathrm{CC}_{1 / 2}$ can be calculated with the $\sigma-\tau$ method, without invoking random selections of observations. The approach to the calculation of $\mathrm{CC}_{1 / 2}$ has a number of advantages compared with the random-selection method using the formula for Pearson's correlation coefficient:

(a) It avoids the numerical spread of results associated with different seeds of the random split assignment and is therefore more accurate. 
(b) It treats odd numbers of reflections consistently, which otherwise lead to unequal numbers in the two half-datasets, which again leads to more accurate results.

(c) It treats the sigma weighting of merged intensities more consistently. The original derivation of properties of $\mathrm{CC}_{1 / 2}$ (Karplus \& Diederichs, 2012, supplement) does not take weighting of intensities into account, whereas our formulation in $\$ 2.3$ naturally accommodates weighted intensities.

(d) The higher precision of $\mathrm{CC}_{1 / 2}$ values allows us to calculate precise $\Delta \mathrm{CC}_{1 / 2}$ values that would vanish in the noise incurred by random assignments.

(e) The calculation of the anomalous $\mathrm{CC}_{1 / 2}\left(\mathrm{CC}_{1 / 2 \_ \text {ano }}\right)$ can be done analogously. For $\mathrm{CC}_{1 / 2 \text { ano, the formula suggests an }}$ answer to a question that has puzzled several crystallographers and was discussed on the CCP4 bulletin board (CCP4 Bulletin Board, 2015): why do we sometimes see negative (mostly anomalous) $\mathrm{CC}_{1 / 2}$ values in high-resolution shells? The formula tells us immediately that this symptom indicates that the average variance of the observations is higher than the variance of the averaged intensities in those particular resolution shells. Obviously, this is consistent with the given situation in which practically no anomalous signal but the usual measurement error is present.

\subsection{Choice of target function and rejection criterion}

There exist two types of errors in crystallographic intensity data: random and systematic. If only random errors are present in the observed intensities $I_{i}$, no datasets should be discarded, no matter how weak they are, since they improve the merged intensities $I_{\text {merged }}$ if the $\sigma_{i}$ are derived from counting statistics. This situation defines 'isomorphism of datasets', in which the following hold:

(i) The relations $I_{\text {merged }}=\sum\left(I_{i} / \sigma_{i}^{2}\right) / \sum\left(1 / \sigma_{i}^{2}\right)$ and $\sigma_{\text {merged }}^{2}=$ $1 / \sum\left(1 / \sigma_{i}^{2}\right)$ hold strictly, and therefore $I_{\text {merged }} / \sigma_{\text {merged }}$ grows monotonically if more data are merged.

(ii) $\mathrm{CC}_{1 / 2}=\left(\sigma_{y}^{2}-\frac{1}{2} \sigma_{\varepsilon}^{2}\right) /\left(\sigma_{y}^{2}+\frac{1}{2} \sigma_{\varepsilon}^{2}\right)$ (as defined in $\S 2$ ) also grows monotonically since the mean error decreases if more data are merged. The value of $\mathrm{CC}^{*}=\left[2 \mathrm{CC}_{1 / 2} /\left(1+\mathrm{CC}_{1 / 2}\right)\right]^{1 / 2}$, itself monotonically depending on $\mathrm{CC}_{1 / 2}$, then is an accurate indicator for the correlation of the merged data and the (unknown) true data (Karplus \& Diederichs, 2012).

However, if systematic errors exist - which is unfortunately always the case, to some extent - these are by definition not independent. The above relations for $I_{\text {merged }}, \sigma_{\text {merged }}$ and $\mathrm{CC}_{1 / 2}$ then tell us the precision, but not the accuracy, of the merged intensities.

The $I_{\text {merged }} / \sigma_{\text {merged }}$ ratio still increases in the presence of systematic errors, since the denominator of $\sum\left(1 / \sigma_{i}^{2}\right)$ grows with every observation merged. $I_{\text {merged }} / \sigma_{\text {merged }}$ is therefore not suitable for identifying systematic error. $\mathrm{CC}_{1 / 2}$, on the other hand, diminishes if data with a sufficient amount of systematic error are merged, since it depends on the agreement of the observed intensity values. Non-isomorphism between datasets is a special case of systematic errors which affect all reflections in a dataset in a way that may in principle be different for every dataset. For simplicity, however, we may assume that most datasets do not differ significantly in systematic ways. This assumption is valid if the crystals are grown from the same protein preparation under the same conditions, the crystals are mounted, measured and processed in the same (or a closely similar) way, and indexing ambiguities (if applicable) have been resolved (Brehm \& Diederichs, 2014).

A single rogue (outlier) dataset then has a small influence on the merged data, but if even the small part of the total dataset that it influences leads to a significant decrease of $\mathrm{CC}_{1 / 2}$, this may be considered a strong hint towards nonisomorphism of this particular dataset, and it appears justified to discard it. Its exclusion should reduce the noise in electron density maps and improve the agreement between merged data and the refined model.

A small degree of non-isomorphism in a dataset may still allow a slight increase or lead to an insignificant decrease of $\mathrm{CC}_{1 / 2}$, such that this dataset cannot be identified with the $\Delta \mathrm{CC}_{1 / 2}$ method. If a large number of such datasets are merged, this will lead to a degradation of the merged intensities, because they introduce into the merged data a mixture of signals corresponding to molecular conformations or states distant from the majority one. Refinement of a single model against such merged data will ultimately also result in elevated $R_{\text {work }} / R_{\text {free }}$ and noise in electron density maps. This means that many slightly non-isomorphous datasets may result in a slight increase of $\mathrm{CC}_{1 / 2}$ while nevertheless decreasing the suitability of the data for refinement.

An increase of $\mathrm{CC}_{1 / 2}$ is thus a necessary but, because of this caveat, not strictly a sufficient condition for improvement of data by merging. In principle, the $\Delta \mathrm{CC}_{1 / 2}$ method shares this restriction with the BLEND method (Foadi et al., 2013), which uses a large cell parameter deviation as rejection criterion. However, since it uses the experimental intensity data, the $\Delta \mathrm{CC}_{1 / 2}$ method directly targets the desired property of optimizing the merged intensity data, and is successful in doing so as seen when being validated. Compared to the pairwisecorrelation method of Giordano et al. (2012), which interprets low correlation as meaning low non-isomorphism, we argue that our method avoids the erroneous rejection of weak datasets, at least in situations where the majority of datasets are isomorphous and a mixture of strong and weak datasets exists.

\subsection{Non-isomorphism in simulated and experimental data}

If datasets are artificially modified such that nonisomorphism is introduced by increasing amounts of unit-cell inflation, a direct relation between $\Delta \mathrm{CC}_{1 / 2 \_i}$ and the amount of unit-cell change is found (Table 2). We find that changes in the unit cell from $0.4 \AA$ can be considered as non-isomorphous for this combination of datasets. This does not mean that nonisomorphism caused by unit-cell changes is in general not detectable below $0.4 \AA$; in fact the threshold is dependent on the resolution of the data and the specific combination of datasets, which is why we propose an iterative usage of the method. 
The most isomorphous dataset is the one with the average of all unit-cell dimensions, which appears to confirm the method of Foadi et al. (2013). The latter method would not have been of much help for the PepT and AlgE projects, however, because their partial datasets have poorly determined unit-cell parameters and therefore our a posteriori analysis could not reveal any particular unit-cell-related deviations or properties of non-isomorphous datasets.

For the latter projects, identification of non-isomorphous datasets was straightforward with the $\Delta \mathrm{CC}_{1 / 2}$ method. Owing to our precise method for $\mathrm{CC}_{1 / 2}$ calculation, outliers may yield high significance levels, and we expect this to hold also for a larger number of datasets.

Unfortunately, from a theoretical point of view it remains unclear which properties the outlier datasets have such that they strongly influence the merged data; further work in this area is underway.

\subsection{Pitfalls of validation}

One way of validating the identification of non-isomorphous datasets would be to refine a model against merged data with and without the dataset in question and to compare $R_{\text {work }} / R_{\text {free }}$ of the two refinements. However, trials to do so convinced us that the small number of free-set reflections present in each partial dataset lead to inconclusive results as $R_{\text {free }}$ showed large variations.

Likewise, direct comparison of squared structure factors from the model with intensities of partial datasets did not lead to conclusive results, since weak datasets displayed low correlations.

We therefore compared, without refinement, $\Delta \mathrm{CC}_{\mathrm{FOC} \_}$, the change in correlation coefficient between observed structure factors and structure factors calculated from their PDB models, with $\Delta \mathrm{CC}_{1 / 2_{i} i}$. In the case of PepT, we found that those datasets which were identified as non-isomorphous according to $\Delta \mathrm{CC}_{1 / 2 \_i}$ also reduce the correlation of the merged data with the model, and thus we confirmed our decision based on $\Delta \mathrm{CC}_{1 / 2 \_i}$. However, the $\mathrm{AlgE}$ datasets displayed the opposite effect, which puzzled us until we realized that the model we were basing the comparison upon had - in contrast to the PepT model we used - originally been refined against the very data we were comparing it with. In other words, the model had been influenced by all datasets, including non-isomorphous ones, and was therefore biased: exclusion of non-isomorphous data from the merged data resulted in an increase of $\Delta \mathrm{CC}_{\mathrm{FOC}_{\imath} i}$. The remedy we found and employed was to add large random shifts with zero mean to the coordinates of the model, thus reducing the bias by forcing the model out of its biased local energy minimum, at the expense of an overall degraded model with little discriminatory power.

One way of avoiding the bias problem would be to perform refinements of a mildly shaken model and leave out each dataset in turn, to arrive at more realistic unbiased $\Delta \mathrm{CC}_{\mathrm{FOC} \_} i$ values. However, this would be a project on its own and was considered outside the scope of this work.

\subsection{Summary}

Our findings demonstrate that non-isomorphous datasets can be identified with an algorithm which uses the numerical value of the average $\mathrm{CC}_{1 / 2}$ across all resolution shells as an optimization criterion. This not only works well with artificial data simulating cell parameter variation but is also demonstrated and validated with experimental data.

Although $\mathrm{CC}_{1 / 2}$ was devised as a precision indicator for the merged data, it can also serve as a proxy for the quality of the model that can be derived from the data, since $\mathrm{CC}_{1 / 2}$ constitutes the link between data and model quality (Karplus \& Diederichs, 2012). This role as proxy is compromised if model bias plays a role. However, contrary to classical model bias where the phases and therefore the electron density map are influenced by the very model that is the goal of structure solution, here we experience a different kind of bias: a dataset influences the model to such an extent that the correlation of model and data always diminishes if that dataset is removed from the merged data (and is strong enough). This leads to the insight that each and every dataset noticeably influences the model, and consequently the model will have to account for all possible constituents and conformations present in the data.

However, systematic differences between crystals cannot properly be modelled in refinement since in serial crystallography the averaging of datasets is incoherently done on intensities, rather than on structure factors as would happen if different conformations occur in the coherently illuminated volume of a single crystal. Since a refined model, with its coherently diffracting constituents, cannot fully approximate a sum of intensities (i.e. squared amplitudes) with a (squared) sum of amplitudes, an elevated level of $R_{\text {work }} / R_{\text {free }}$ and noise in electron density maps would result if non-isomorphism is not detected and the worst datasets are not excluded.

In our trials with experimental data measured at a synchrotron, the outcome was the rejection of only a single (as for PepT and AlgE) or a few rogue datasets. However, this rather attests to the consistent quality of the data obtained in SSX. Finally, we note that our procedure is equally applicable to datasets obtained from SFX. Rejection rates may be higher in the latter method since its technology is less mature than that of SSX and the absolute numbers of datasets are high. We therefore expect that the $\Delta \mathrm{CC}_{1 / 2}$ method will be useful in SFX.

\section{Acknowledgements}

We are greatly indebted to Martin Caffrey and his group (Trinity College, Dublin, Ireland), as well as to Meitian Wang and his group (Paul-Scherrer-Institute, Villigen, Switzerland), for allowing us to use the PepT and $\mathrm{AlgE}$ datasets. The authors gratefully acknowledge funding and support by the Konstanz Research School Chemical Biology.

\section{References}

Adams, P. D., Grosse-Kunstleve, R. W., Hung, L.-W., Ioerger, T. R., McCoy, A. J., Moriarty, N. W., Read, R. J., Sacchettini, J. C., Sauter, N. K. \& Terwilliger, T. C. (2002). Acta Cryst. D58, 1948-1954. 
Blundell, T. L. \& Johnson, L. N. (1976). Protein Crystallography. New York: Academic Press.

Brehm, W. \& Diederichs, K. (2014). Acta Cryst. D70, 101-109.

CCP4 Bulletin Board (2015). Thread 'Negative CCanom'; 25 messages between 16 July and 23 July 2015, retrieved from https://www.jiscmail.ac.uk/cgi-bin/webadmin? A1=ind1507\&L= CCP4BB\&X=B527CF01A7EA79AD76\#51.

Chapman, H. N. et al. (2011). Nature, 470, 73-77.

Darwin, C. G. (1914). Philos. Mag. Ser. 6, 27, 315-333.

Darwin, C. G. (1922). Philos. Mag. Ser. 6, 43, 800-829.

Dickerson, R. E., Kendrew, J. C. \& Strandberg, B. E. (1961). Acta Cryst. 14, 1188-1195.

Diederichs, K. (2009). Acta Cryst. D65, 535-542.

Diederichs, K. \& Karplus, P. A. (2013). Acta Cryst. D69, 12151222.

Foadi, J., Aller, P., Alguel, Y., Cameron, A., Axford, D., Owen, R. L., Armour, W., Waterman, D. G., Iwata, S. \& Evans, G. (2013). Acta Cryst. D69, 1617-1632.

Giordano, R., Leal, R. M. F., Bourenkov, G. P., McSweeney, S. \& Popov, A. N. (2012). Acta Cryst. D68, 649-658.
Huang, C.-Y., Olieric, V., Ma, P., Howe, N., Vogeley, L., Liu, X., Warshamanage, R., Weinert, T., Panepucci, E., Kobilka, B., Diederichs, K., Wang, M. \& Caffrey, M. (2016). Acta Cryst. D72, 93-112.

Huang, C.-Y., Olieric, V., Ma, P., Panepucci, E., Diederichs, K., Wang, M. \& Caffrey, M. (2015). Acta Cryst. D71, 1238-1256.

Kabsch, W. (2010a). Acta Cryst. D66, 125-132.

Kabsch, W. (2010b). Acta Cryst. D66, 133-144.

Karplus, P. A. \& Diederichs, K. (2012). Science, 336, 1030-1033.

Karplus, P. A. \& Diederichs, K. (2015). Curr. Opin. Struct. Biol. 34, 60-68.

Kendrew, J. C., Dickerson, R. E., Strandberg, B. E., Hart, R. G., Davies, D. R., Phillips, D. C. \& Shore, V. C. (1960). Nature, 185, 422427.

Kleywegt, G. J. (1995). CCP4/ESF-EACBM Newsl. Protein Crystallogr. 31, 45-50.

Liu, Q., Liu, Q. \& Hendrickson, W. A. (2013). Acta Cryst. D69, 13141332.

Nanao, M. H., Sheldrick, G. M. \& Ravelli, R. B. G. (2005). Acta Cryst. D61, 1227-1237.

Rossmann, M. G. (2014). IUCrJ, 1, 84-86. 